$\mathbf{E}^{\mathrm{n}}$ n las zonas áridas, el establecimiento de nuevos individuos por la vía sexual es poco frecuente debido a las condiciones extremas de temperatura y disponibilidad de agua. En cambio, la propagación vegetativa es común y ecológicamente importante como estrategia de invasión y establecimiento rápido (Palleiro, 2001). De hecho, en algunos taxa xerófilos donde el reclutamiento sexual es bajo o está ausente, la propagación vegetativa puede ser suficiente para mantener una población (Mandujano, 1998).

Las estructuras vegetativas como tallos, bulbos, rizomas y esquejes tienen mayores probabilidades de establecimiento que las plántulas procedentes de semilla. Por un lado, las estructuras vegetativas provienen de genotipos probados de plantas ya establecidas y sólo tienen que continuar con su crecimiento y desarrollo. Además, tales estructuras poseen una gran cantidad de recursos al ser mayores que las semillas, y no se tardan tanto en crecer o establecerse. Finalmente, las estructuras vegetativas son menos susceptibles a la depredación que las plántulas, las cuales son de menor tamaño (Mandujano, 1998; Palleiro, 2001; Arizaga y Ezcurra, 2002).

Se ha reportado que diversas especies de Opuntia se reproducen principal o exclusivamente por propagación vegetativa (Mandujano et al., 1996). En el caso de Cylindropuntia leptocaulis (DC.) F.M.Knuth (Cactaceae) se observa que además de la reproducción sexual mediante semillas se presenta propagación vegetativa a partir de frutos prolíficos que desarrollan ramas (figura 1). Esto es posible puesto que en este género el ovario se encuentra inmerso dentro de un tallo modificado, o pericarpelo, el cual cuenta con entrenudos (areolas) en donde hay tejido meristemático activo (Bravo-Hollis, 1978). Las ramas podrían favorecer el estableci-

\section{LA PRODUCCIÓN DE FRUTOS PROLÍFICOS EN CYLINDROPUNTIA LEPTOCAULIS (CACTACEAE) COMO UNA ALTERNATIVA ANTE EL FRACASO
DE LA REPRODUCCIÓN SEXUAL}

\author{
Erika F. VÁzQuez-Delfín, Sinthia L. Sánchez-Serrano y \\ Carlos Martorell ${ }^{1}$
}

'Departamento de Ecología y Recursos Naturales, Facultad de Ciencias, Universidad Nacional Autónoma de México. Tel (55) 5622-4835, Fax (55) 5622-4828, correo-e: martorel@miranda.ecologia.unam.mx

miento de los frutos que las desarrollan. A lo largo de varios años y en diferentes épocas del año hemos observado frutos enraizados en el suelo, pero en todos los casos se ha tratado de frutos ramificados. Nunca hemos visto que aquellos carentes de ramas se hayan establecido en forma natural. Esta forma de propagación vegetativa puede representar una vía para "rescatar" el gasto energético que se ha efectuado en la producción de un ovario de grandes dimensiones. Este fenómeno podría ser equivalente al que se ha observado en el género Agave, donde, una vez formado el escapo, la planta desarrolla bulbilos cuando la producción de frutos fracasa (Arizaga y Ezcurra, 1995).

En el presente estudio se determinó si existe una relación entre la producción de semillas y de ramas en el fruto, con el fin de conocer si una baja producción de semillas puede ser compensada con el desarrollo de ramas que le permitan al fruto

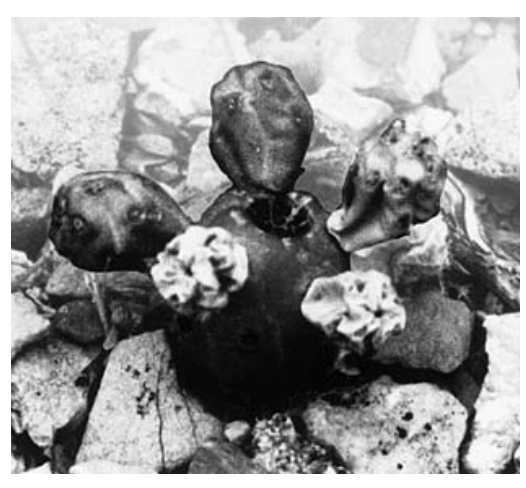

Figura 1. Un fruto prolífico enraizado de Cylindropuntia leptocaulis. Fotografía: Diana Mota.

establecerse, funcionando como un seguro contra el fracaso reproductivo o incluso sustituyendo la reproducción sexual.

El estudio se llevó a cabo en Peña Blanca, Querétaro, México, en un matorral xerófilo dominado por Larrea tridentata y Acacia constricta, a una altitud aproximada de $1,500 \mathrm{~m}$ y una latitud de $21^{\circ} \mathrm{N}$. La precipitación 
anual en la estación meteorológica de Peñamiller, ubicada a unos $15 \mathrm{~km}$ del sitio de estudio, es de $456.3 \mathrm{~mm}$ y la temperatura media anual es de $21.7^{\circ} \mathrm{C}$ (García, 1987).

Cylindropuntia leptocaulis es un arbusto bajo de 1 a $1.5 \mathrm{~m}$ de altura. Posee un tronco leñoso bien definido con ramas cilíndricas. La flores son generalmente hermafroditas y el fruto es carnoso, globoso, presenta glóquidas y algunas veces es prolífico (Bravo-Hollis, 1978).

Se colectaron 110 frutos y se cuantificó el número de semillas y el número de ramas en cada uno de ellos. Mediante una regresión loglineal (McCullagh y Nelder, 1983) se determinó que el número de ramificaciones tiende a aumentar significativamente conforme disminuye el número de semillas en el fruto de Cylindropuntia leptocaulis $\left(\chi^{2}=\right.$ $12.83, P=0.0003$; figura 2 ). El dato de la extrema derecha en la figura 2 parece tener un peso excesivo en la regresión, pero aunque sea eliminado ésta sigue siendo significativa $\left(\chi^{2}=\right.$ $6.95, P=0.0083$ ).

A pesar de que se observó que la mayoría de los ovarios cuentan con ocho óvulos, el número de frutos encontrados con más de cinco semillas fue mínimo. Esto sugiere que la reproducción sexual sufre algún tipo de limitación, ya sea por falta de polinizadores, depresión endogámica o limitantes en el presupuesto energético debidas a condiciones estresantes tales como la escasez de agua, el tipo de sustrato y las altas temperaturas (Mandujano et al., 1996; Palleiro, 2001; Plasencia, 2003). Esta situación podría representar una presión selectiva que tal vez haya promovido el desarrollo de frutos prolíficos como alternativa a la reproducción sexual en la transferencia de genes a las siguientes generaciones.

La relación negativa encontrada en este trabajo entre las variables analizadas sugiere que en

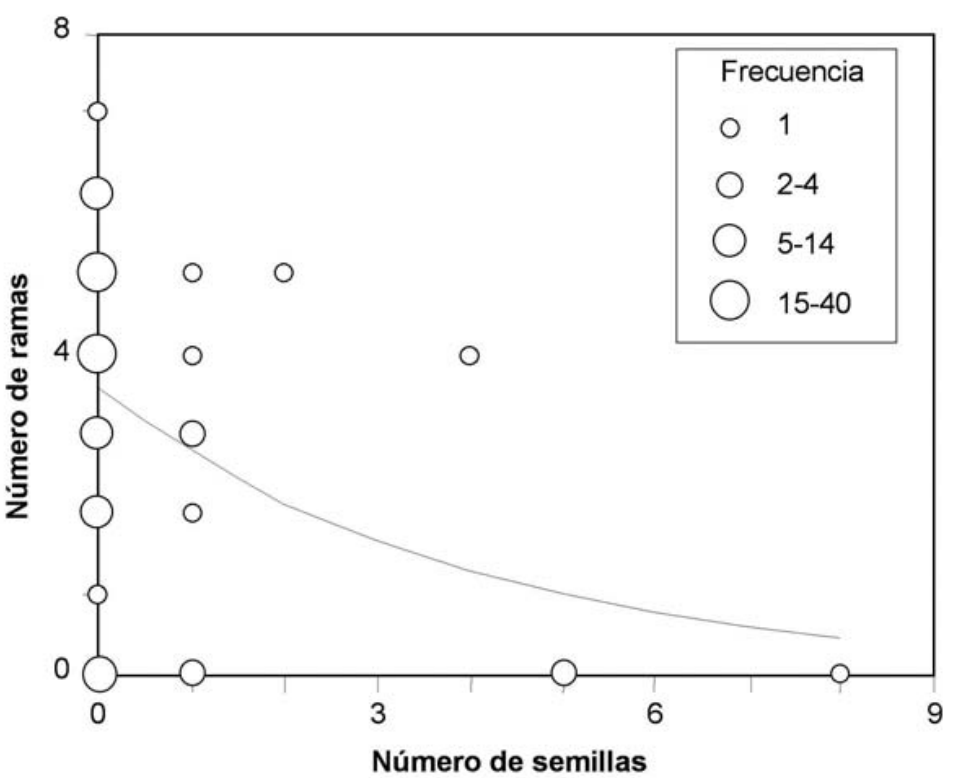

Figura 2. Relación entre el número de semillas y de ramas en los frutos de Cylindropuntia leptocaulis. El tamaño de los círculos indica el número de datos observados.

Cylindropuntia leptocaulis la baja producción de semillas resulta en el desarrollo de ramas en el fruto. Las ramas pueden funcionar como reservas de agua o energéticas, o bien proveer al fruto de una mayor superficie fotosintéticamente activa que le permita nutrirse y desarrollarse. De tal forma, el fruto prolífico puede funcionar como una estructura vegetativa con una mayor probabilidad de establecimiento, tal como sucede en bulbilos producidos por Agave macroacantha (Arizaga y Ezcurra, 2002). Ambas estructuras, los bulbilos y los frutos prolíficos, son semejantes en cuanto a que son propágulos que, por su desarrollo, son capaces de desempeñar todas las funciones de un individuo independiente y de establecerse al caer al suelo.

Las consecuencias de la formación de frutos prolíficos plantean nuevas interrogantes. Desde un punto de vista demográfico queda por determinar si esta alternativa a la reproducción sexual es suficiente para mantener a las poblaciones silvestres, así como el destino en el mediano plazo de los frutos que logran enraizar. También resulta atractivo el estudio del fenómeno con el enfoque de la evolución de historias de vida, evaluando los costos y beneficios de las diferentes formas de propagación de la especie, así como de la inversión en cuidado paternal que efectúa la planta madre, ya que es poco probable que la fotosíntesis que se realiza en el pericarpelo sea suficiente para subsidiar la formación de nuevas ramas.

El desarrollo de frutos prolíficos en Cylindropuntia leptocaulis es una característica que funciona como un seguro contra el fracaso en la producción de semillas en un ambiente donde comúnmente la reproducción sexual es poco exitosa. Esta es la tercera especie en la que se ha reportado la modificación de las estructuras de reproducción sexual para la propagación vegetativa, y la primera fuera de la familia Agavaceae. 


\section{Agradecimientos}

De manera particular a Teresa Valverde y Alejandro Zavala, quienes hicieron comentarios valiosos sobre versiones preliminares del manuscrito. Agradecemos a los integrantes del grupo 5154 de Ecología I por su ayuda en el campo y a la Facultad de Ciencias - Universidad Nacional Autónoma de México por el apoyo logístico.

\section{Literatura citada}

Arizaga S. y Ezcurra E. 1995. Insurance against reproductive failure in a semelparous plant: bulbil formation in Agave macroacantha flowering stalks. Oecologia 101:329-334.

Arizaga S. y Ezcurra E. 2002. Propagation mechanisms in Agave macroacantha (Agavaceae), a tropical arid-land succulent rosette. American Journal of Botany 89:632641.

Bravo-Hollis H. 1978. Las Cactáceas de México. Vol. 1. Universidad Nacional Autónoma de México, México, D.F.

García E. 1987. Modificaciones al Sistema de Clasificación Climática de Köppen. $4^{\mathrm{a}}$ edición. Edición de la autora, México, D.F.

Mandujano M.C. 1998. The relative contributions of sexual reproduction and clonal propagation in Opuntia rastrera from two habitats in the Chihuahuan desert. Journal of Ecology 86:911921.

Mandujano M.C., Montaña C. y Eguiarte L.E. 1996. Reproductive ecology and inbreeding depression in Opuntia rastrera (Cactaceae) in the Chihuahuan desert: why are sexually derived recruitments so rare? American Journal of Botany 83:63-70.
McCullagh P. y Nelder J.A. 1983. Generalized Linear Models. Chapman $\&$ Hall, Londres.

Palleiro N. 2001. Propagación vegetativa a través de frutos abortados de Opuntia microdasys en el desierto chihuahuense. Tesis profesional, Facultad de Ciencias, Universidad Nacional Autónoma de México, México, D.F. 75 pp.

Plasencia L. 2003. Biología reproductiva de Opuntia bradtiana (Cactaceae) en Cuatro Ciénegas, Coahuila. Tesis profesional, Facultad de Ciencias, Universidad Nacional Autónoma de México, México, D.F. 49 pp.

Fecha de recepción: 5 de octubre de 2004

Versión corregida: 26 de enero 2005 Aceptado: 26 de enero 2005 